\title{
Using Neural Networks to Predict the Hardness of Aluminum Alloys
}

\author{
Bilal M. Zahran \\ Department of Computer Engineering \\ Faculty of Engineering Technology \\ Al-Balqaa Applied University, Jordan \\ zahranb@yahoo.com
}

\begin{abstract}
Aluminum alloys have gained significant industrial importance being involved in many of the light and heavy industries and especially in aerospace engineering. The mechanical properties of aluminum alloys are defined by a number of principal microstructural features. Conventional mathematical models of these properties are sometimes very complex to be analytically calculated. In this paper, a neural network model is used to predict the correlations between the hardness of aluminum alloys in relation to certain alloying elements. A backpropagation neural network is trained using a thorough dataset. The impact of certain elements is documented and an optimum structure is proposed.
\end{abstract}

Keywords- aluminum alloys; hardness; neural networks

\section{INTRODUCTION}

Aluminum is a light metal exhibiting a high degree of corrosion resistance in normal corrosive environments [1]. Aluminum is almost always alloyed, which significantly improves its mechanical properties [1, 2]. The main alloying elements are copper (Cu), zinc ( $\mathrm{Zn})$, magnesium $(\mathrm{Mg})$, manganese $(\mathrm{Mn})$, iron (Fe) and silicon (Si) [3]. A properly treated aluminum alloy may possess a strength level about thirty times that of pure aluminum [1, 3]. The properties of a particular aluminum product depend on the alloy chosen. The mechanical properties of aluminum alloys are defined by a number of principal microstructural features [2]. Hardness is a mechanical property that measures the ability of a material to resist indentation and it is closely related to the material's strength [1].

Neural networks (NNs) are non-linear mapping structures inspired by the function of the human brain. They are considered powerful modeling tools especially for data with unknown underlying relationships. Neural networks have been widely used in prediction problems of mechanical properties of materials [6-8]. NNs consist of computational elements called neurons, operating in parallel, connected by links with variable weights which are typically adapted during the learning process $[4,5]$. Feed forward multilayer networks trained with the backpropagation algorithm are probably the most common kind. In this paper the relation between the hardness of aluminum alloys and the percentage of alloying elements was predicted using such NNs, through several scenarios.

\section{ALUMINUM AND ITS ALLOYS}

Aluminum and its alloys are rather popular in the construction, marine and aircraft industries because of its ease of fabrication, non-toxicity, strength (pound-for pound), and resistance to the corrosive atmospheres of industry and marine environments [1, 3]. Aluminum and its alloys are divided in two categories, cast and wrought, and each group is further subdivided into classes according to the composition. Every aluminum alloy has its own properties and characteristics which distinct it from the other aluminum alloys, and every aluminum alloy is consisted of a number of alloying elements in different percentages. Each alloying element has its particular effect to the alloy's properties such are electrical conductivity, thermal conductivity, corrosion resistance, thermal expansion, melting temperature, strength yield and hardness. A thorough dataset was employed for this research and Table I shows a sample of the dataset used.

TABLE I. SAMPLE OF THE DATASET FOR THE ALUMINUM ALLOYS

\begin{tabular}{|c|c|c|c|c|c|c|}
\hline \multirow{2}{*}{ Alloy number } & \multicolumn{5}{|c|}{ Elements percentages (wt \%) } & \multirow{2}{*}{ Hardness } \\
\cline { 2 - 6 } & $\mathbf{S i}$ & $\mathbf{F e}$ & $\mathbf{M n}$ & $\mathbf{M g}$ & $\mathbf{C u}$ & \\
\hline 1 & 0.04 & 0.04 & 0.3 & 0.29 & 4.6 & 70 \\
\hline 2 & 0.03 & 0.04 & 0.3 & 0.28 & 4.6 & 76 \\
\hline 3 & 0.03 & 0.04 & 0.3 & 0.28 & 4.6 & 77 \\
\hline 4 & 0.04 & 0.04 & 0.3 & 0.29 & 4.7 & 67 \\
\hline 5 & 7.2 & 0.125 & 0.0026 & 0.35 & 0.1 & 59 \\
\hline 6 & 7 & 0 & 0 & 0.3 & 0 & 79 \\
\hline 7 & 0.195 & 0.418 & 0.588 & 1.12 & 0.122 & 80.2 \\
\hline 8 & 5.902 & 0 & 0 & 0.461 & 0.007 & 88 \\
\hline 9 & 0.24 & 0.28 & 0.4 & 2 & 4.75 & 90 \\
\hline 10 & 0 & 0 & 0 & 0 & 0 & 69.5 \\
\hline
\end{tabular}

Hardness tests are a useful tool for the evaluation of materials and the quality control of manufacturing processes in research and development work. It gives an indication of a material's properties, such as strength, ductility and wears resistance. There is a wide range of different hardness tests. Each test gives a different value for hardness. Therefore, hardness is not a universal well-defined materials property, but 
depends on the method used for measurement. One of the most popular methods was used in this research (Brinell hardness). The method relies on measuring the size of an impression left by an indenter under a known load [1, 3].

\section{NEURAL NETWORKS AND THE PROPOSED METHOD}

A typical neuron has three basic parts: input weights, a soma, and an activation function [4, 5]. The input weights scale the values used as inputs to the neuron, the soma adds all the scaled values together, and the activation function produces the final output of the neuron. The backpropagation (BP) algorithm is one of the most famous training algorithms for multilayer neural networks. BP is a gradient descent technique to minimize the error $E$ for a particular training pattern $[4,5]$. Several researches have been conducted on predicting some properties of materials, machining and other fields through the use of neural networks [6-12].

The initial steps followed in developing the NN used in this paper was the choice of architecture (3-layer $\mathrm{NN}$ ), the random initialization of weighs and the definition of defining the number of neurons for the hidden layer. The alloying elements percentage in the aluminum alloys were used as input data for the NNs and the hardness value was selected as the target output. Before training, the dataset was randomly divided into two groups. $75 \%$ of the data were used for training and $25 \%$ for testing. At the start of the training phase, the NN was activated with several input vectors, and the output was calculated. The calculated outputs were compared to the target values to produce the error values. According to the magnitude of the error, each weight was adjusted so as to reduce the total error. Then the calculation was performed once again using the adjusted weights in order to get a new set of outputs. These in turn were compared with the targets, and the weights were adjusted again. This process was repeated until an acceptable criterion was reached.

For the experiments in this paper, the total error (i.e. error based on the mean squared error (MSE) between the network output and target), was used as a criterion for terminating the training session. The model reached at the end of the training phase was used in the testing phase. During the testing phase, the trained neural network was tested with the data specified for this purpose, and the output was calculated. Its performance was evaluated using three performance measures: the mean square error (mse), prediction error (pe) and mean absolute error (mae).

\section{RESUlTS AND DISCUSSION}

The NN model is used to predict correlations between a mechanical property (hardness) of the aluminum alloys with the alloying elements. More than 72 experiments on the dataset were conducted. During the development process, three parameters were tested for suitability: the number of neurons in the hidden layer, the learning rate and the activation function.

Every time one parameter was kept constant and the others were changed in order to reach the best value for the parameters. The error was calculated and the weights were updated after each input/output pair was presented. In order to investigate the effect of the number of neurons on the prediction results, a trial-and-error procedure with a variable number of neurons in the hidden layer was applied. The BPNN structure of one hidden layer with a learning rate of 0.4 and a momentum rate of 0.6 was trained using various numbers of hidden neurons which varied from 3 to 15 . It was seen that a network with 6 neurons in the hidden layer provided the best mapping and, therefore, an architecture of 6 hidden neurons along with one hidden layer (variable-6-1) was chosen. After that, different activation functions for hidden and output layers were tested. The activation functions tested were log-sogmoid, linear and hyperbolic tangent sigmoid. Table II shows the results when changing the activation functions and while fixing other parameters.

TABLE II. THE PERFORMANCE FOR DIFFERENT ACTIVATION FUNCTIONS

\begin{tabular}{|c|c|c|c|}
\hline $\begin{array}{c}\text { Input Parameter: } \\
\text { Activation function }\end{array}$ & log sigmoid & linear & $\begin{array}{c}\text { hyperbolic tangent } \\
\text { sigmoid }\end{array}$ \\
\hline mse & 0.033 & 0.076 & 0.080 \\
\hline mae & 0.8 & 3 & 1.6 \\
\hline pe & 2.2 & 3.2 & 2.5 \\
\hline
\end{tabular}

The best activation function was found to be the log sigmoid for both input and output layers. The learning and regression curves of the best model are plotted in Figure 1 and Figure 2 respectively. Furthermore, Table III shows a sample of the output of the NN model compared with the target outputs. This is achieved when the number of inputs equals 5, the number of neurons in the hidden layer equals 6 , the learning rate equals 0.4 and the activation function was the "log sigmoid” for both input and output layers.

TABLE III. SAMPLE OF THE TARGET AND PREDICTED VALUES OF HARDNESS

\begin{tabular}{|c|c|}
\hline Target values of hardness & Predicted values of hardness \\
\hline 90.000 & 93.984 \\
\hline 88.000 & 91.592 \\
\hline 80.200 & 87.712 \\
\hline 79.000 & 82.206 \\
\hline 77.000 & 81.984 \\
\hline 76.000 & 79.984 \\
\hline 70.000 & 83.984 \\
\hline 69.500 & 79.635 \\
\hline 67.000 & 73.984 \\
\hline 59.000 & 66.578 \\
\hline
\end{tabular}

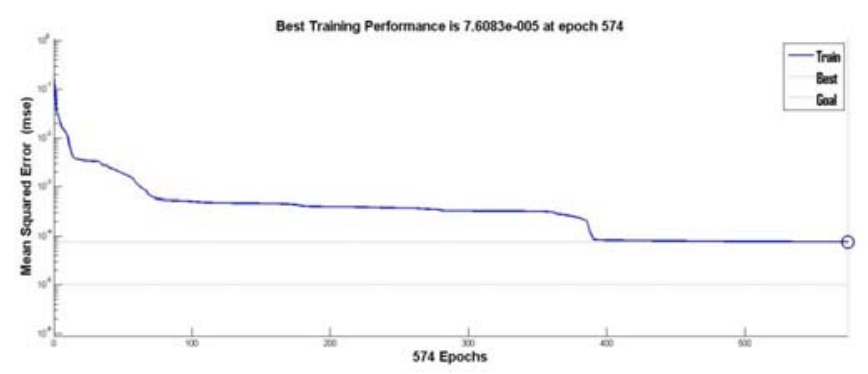

Fig. 1. Learning curve for the neural network best model. 


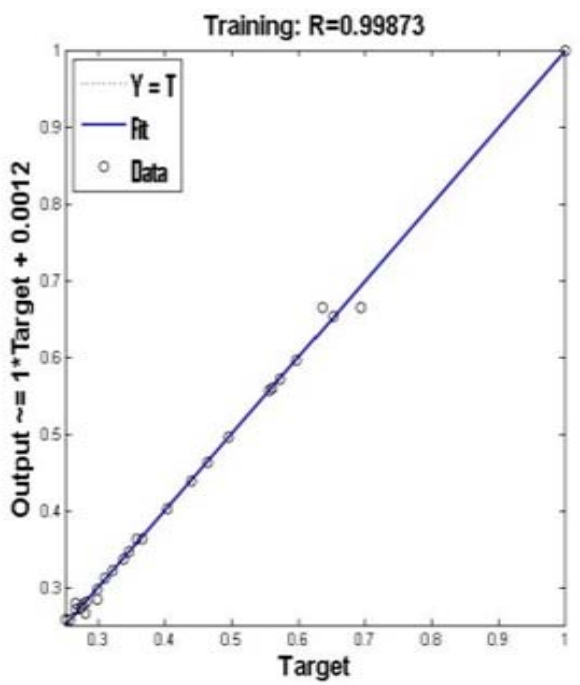

Fig. 2. The regression curve of the NN best model.

After testing several different combinations of the alloying elements in the experiments, the following were documented:

- The elements that individually increase the hardness of the alloy the most are $\mathrm{Fe}$ and $\mathrm{Cu}$.

- The elements that individually have no significant effect on the hardness of the alloy are $\mathrm{Mg}, \mathrm{Mn}$ and $\mathrm{Si}$.

- $\quad$ The structure [Cu, Fe, Mn and Si] in alloys gave the best result regarding hardness.

\section{CONCLUSIONS}

The main purpose of this paper is to investigate the relationship between hardness and the alloying elements percentages using neural networks. An optimum architecture was achieved through various tests. The NN model predicted the hardness of aluminum alloys efficiently. The impact of different individual elements on the hardness was calculated and a specific structure was found to provide the best results regarding hardness.

\section{REFERENCES}

[1] D. G. Altenpohl, Aluminum: Technology, Applications and Environment: A Profile of a Modern Metal Aluminum from Within, 6th Edition, Wiley, 2010

[2] R. S. Rana, R. Purohit, S. Das, "Reviews on the Influences of Alloying elements on the Microstructure and Mechanical Properties of Aluminum alloys and aluminum alloy composites", International Journal of Scientific and Research Publications, Vol. 2, No. 6, pp. 1-7, 2012

[3] L. Mondolfo, Aluminum alloys: structure and properties, Butterworths, 1976

[4] T. M. Mitchell, Machine Learning, McGraw-Hill, 1997

[5] D. W. Patterson, Artificial Neural networks: Theory and Application, Prentice Hall, 1996

[6] E.Alibeiki, J. Rajabi, J. Rajabi, "Prediction of mechanical properties of to heat treatment by Artificial Neural Networks", Journal of Asian Scientific Research, Vol. 2, No. 11, pp. 742-746, 2012

[7] S. K. Das, S. Kumari, “A Multi-Input Multi-Output Neural Network Model To Characterize Mechanical Properties Of Strip Rolled High Strength Low Alloy (HSLA) Steel”, MS'10 Prague Proceedings of the
International Conference on Modelling and Simulation, Prague, Czech Republic, June 22-25, 2010

[8] X. Liujie, X. Jiandong, W. Shizhong, Z. Songmin, Z. Yongzhen, L. Rui, "Use of Artificial Neural Network in Predicting Mechanical Properties of High-Speed Steel (HSS)”, Proceedings of the 2006 IEEE International Conference on Mechatronics and Automation, pp. 1872-1877, Luoyang, Henan, China, 2006

[9] F. Musharavati, A. S. M. Hamouda, "Application of artificial neural networks for modelling correlations in age hardenable Aluminum alloys”, Journal of Achievements in Materials and Manufacturing Engineering, Vol. 41, No. 1-2, pp. 140-146, 2010

[10] J. H. Su, Q. M. Dong, P. Liu, H. J. Li, B. X. Kang, "Simulation of aging process of lead frame copper alloy by an artificial neural network”, Transactions of Nonferrous Metals Society Of China, Vol.13, No. 6, pp. 1419-1423, 2003

[11] A. Kermanpur, A. Ebnonnasir, A. R. K. Yeganeh, J. Izadi, “Artificial Neural Network Modeling of High Pressure Descaling Operation in Hot Strip Rolling of Steels”, ISIJ International, Vol. 48, No. 7, pp. 963-970, 2008

[12] M. B. Esfahani, M. R. Toroghinejad, S. Abbasi, "Artificial Neural Network Modeling the Tensile Strength of Hot Strip Mill Products”, ISIJ International, Vol. 49, No. 10, pp. 1583-1587, 2009

\section{AUTHOR PROFILE}

Bilal Zahran received the B.Sc degree in Electrical \& Electronic Eng. from the Middle East Technical University, Turkey, in 1996, the M.Sc degree in Communications Eng. from the University of Jordan, Jordan, in 1999, and the $\mathrm{PhD}$ degree in Computer Information System (CIS) from the Arab Academy for Banking and Financial Sciences, Jordan, in 2009. He is currently working as an Assistant Professor at the department of Computer Engineering, Faculty of Engineering Technology, Al-Balqa Applied University, Jordan. His research interests include machine learning, data mining and optimization fields. 